\author{
Military Technical College \\ Kobry El-kobbah, \\ Cairo, Egypt
}

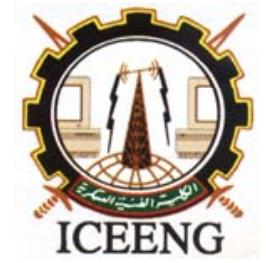

\title{
JOINT DEINTERLEAVING/IDENTIFICATION OF RADAR PULSES USING MATRIX DIFFERENCES
}

Hossam E. Abou-Bakr Hassan $^{*}$ and Khaled H. Moustafa ${ }^{*}$

\begin{abstract}
A radar Electronic Support Measures (ESM) system performs two functions, warning of impending emitters and surveillance to determine the radar activities. Automatic radar ESM system consists of a passive radar receiver which receives emissions from other platforms, measures the parameters of each received pulse and a deinterleaver that sorts the intercepted pulses to enable determination of the individual radar parameters. Theses parameters are compared with the stored parameters of known radars to identify the intercepted emitter type. Consequently the threat level and the optimum jamming program for the intercepted emitter could be derived. This paper proposes a new approach to deinterleave the intercepted pulses and identify their corresponding radars in one step. The proposed approach can successfully identify radars whose angles of arrival are very close. Moreover, the proposed approach can be applied as an integral part of the adaptive deinterleaving algorithm to prevent the ESM from taking actions against false radars and consequently, avoids a waste of the available resources. Computer simulation results have shown that the proposed approach can successfully deinterleave radar pulses and identify their corresponding radars.
\end{abstract}

\section{KEYWORD}

Passive radar, deinterleaving, ESM (Electronic Support Measures) system.

\section{INTRODUCTION}

In a dense emitter environment encountered in modern warfare, the ESM system will receive a seemingly random pulse stream consisting of interleaved pulse chains. In order to identify the intercepted radars, their pulses must be segregated. Broadly speaking, an automatic ESM system consists of three subsystems [1]. The ESM receiver, which is a passive radar receiver, picks up the pulses transmitted by various radars in the environment and measures their individual parameters. These parameters are angle of arrival (AOA), radio frequency (RF), pulse width (PW), pulse amplitude (PA) and time of arrival (TOA). The measured parameters of every successfully intercepted pulse are encoded in a digital format called the pulse descriptor vector (PDV). The deinterleaver sorts the PDVs and forms pulse cells each comprising a set of PDVs assumed to belong to the same emitter. Each pulse cell is assigned

\footnotetext{
${ }^{*}$ Egyptian Armed Forces
} 
an emitter descriptor vector (EDV) whose components are the emitter characteristics such as AOA, RF, PW, pulse repetition interval (PRI), agility and scan period. The main processor compares the EDVs with others stored in the threat library of the EW system to identify the intercepted radars.

Generally, deinterleaving algorithms are classified depending on whether they use the parameters of more than one pulse such as the PRI [2-4], or they use the parameters of a single pulse such as AOA, RF, and PW [5]. The first type of deinterleaving algorithms is denoted as interval-only algorithm, while the other type is denoted as multiple-parameter deinterleaving algorithm. The multiple-parameter deinterleaving algorithm [5] will improve the reliability and the processing speed, compared with the interval-only algorithm. This is expected since it processes only one pulse at a time whereas the algorithms presented in [2-4] are applied to all received pulses during a given observation interval. The multiple-parameter deinterleaving algorithm [5] sorts the input pulses into a number of radar cells based on their mono-pulse parameters. Then, an interval-only algorithm could be used to analyze each individual cell. In this situation applying the interval-only algorithms to each individual cell will be much faster. However, with the existence of complex radars like jittered or staggered PRI and the frequency agile radars, the multiple-parameter deinterleaving algorithm [5] may generate some false cells. The adaptive deinterleaving algorithm [6,7] overcomes this problem by (1) relying only on the AOA to segregate the intercepted pulses, because AOA is a pulse parameter that is difficult for any emitter to change in a very short time, (2) evaluating the quality of each deinterleaved cell by a confidence level $[6,7]$. Only radar cells having a high confidence level will be submitted to the threat library for identification. This is to conserve the limited resources of the EW system that can be used against the false radars.

In modern warfare, some radars may have AOAs close to each other and the pulses emitted from these radars will be merged and constitute a single cell. Thus, the confidence level that represents the quality of this cell $[6,7]$ will be low and consequently, this cell will not be submitted to the threat library for identification. Therefore, in this paper a new approach is proposed to identify radars in low confidence level cells. With this approach, the deinterleaving of the intercepted pulses and the identification of the corresponding radars are accomplished simultaneously. Computer simulations are used to verify the validity of the proposed method.

This paper contains 6 sections. Section 1 is an introduction. Section 2 presents the adaptive deinterleaving algorithm. In this algorithm the quality of each estimated radar cell is evaluated. Section 3 presents a novel approach of joint deinterleaving/identification to solve the difficult problem that may face the ESM system in the dense electromagnetic environment, where hostile radars may have angle of arrivals (AOAs) close to each other and the pulses from these radars are merged and constitute a single cell. Consequently, the quality of that cell will be low, and will not be considered valid. Section 4 presents a method that used to extract the pulses corresponding to the already identified radars from the received stream to simplify the identification of the remaining radars in the received stream. Simulation results are presented in Section 5 to verify that the proposed approach can successfully deinterleave radar pulses and identify their corresponding radars. Section 6 presents the conclusions and the main contributions of the paper. 


\section{ADAPTIVE DEINTERLEAVING ALGORITHM}

The adaptive deinterleaving algorithm [7] is performed in two steps. In the first step, the intercepted pulses are sorted into separate radar cells based on their measured parameters. In the second step a confidence level is evaluated for each deinterleaved cell based on the TOA of the pulses in that cell. Only radar cells having a high confidence level will be submitted to the threat library for identification. This is to conserve the limited resources of the EW system that can be used against the false radars. Pulses of cells with a high confidence level are extracted from the received pulse sequence and the above two steps are again performed for the resulting pulse sequence until no cell has a high confidence level.

The AOA of the intercepted pulses is considered as the most stationary deinterleaving parameter $[1,3]$. The RF is chosen as the second deinterleaving parameter $[1,3]$. However, frequency-agile radars could cause a large number of radar cells representing the same radar. The PW is an unreliable deinterleaving parameter [1,3] because of two reasons. The first reason is the time overlapping of radar pulses in dense emitter environment. The second reason is the effect of the multi-path propagation, which distorts the pulse shape. Thus, the PW and the RF are unreliable deinterleaving parameters and we have to rely only on the AOA to deinterleave the intercepted pulses. The $\mathrm{i}^{\text {th }}$ received pulse will fall in the domain of the $\mathrm{k}^{\text {th }}$ cell depending on the metric coefficient between cell $\mathrm{k}$ and pulse $\mathrm{i}$ which is calculated from the following equation [7]

$$
M_{K, i}=\frac{\left(A O A_{K}-A O A_{i}\right)^{2}}{(2 \triangle A O A)^{2}}
$$

where $\mathrm{AOA}_{\mathrm{k}}$ is the average $\mathrm{AOA}$ of the $\mathrm{k}^{\text {th }}$ cell, $\mathrm{AOA}_{\mathrm{i}}$ is the $\mathrm{AOA}$ of the $\mathrm{i}^{\text {th }}$ incoming pulse and $2 \triangle \mathrm{AOA}$ is the maximum permissible mismatch in AOA. The maximum permissible mismatch in AOA is assumed to be double the measurement accuracy of the AOA. A confidence level [7] is calculated for each deinterleaved cell by analyzing the TOA of the pulses in that cell. Inside each estimated cell, the difference between the TOA of the $i^{\text {th }}$ pulse and the TOA of the $(i+1)^{\text {th }}$ pulse is defined as $\Delta_{\mathrm{i}}$. Fig. 1 illustrates the $\Delta$-histograms of radars with different PRI modes. The histograms in Fig. 1 represent the $\Delta$-histograms of the deinterleaved cells at the output of an ideal deinterleaver. However, in a real situation, a number of pulses may be missing from an estimated radar cell or pulses from different radars are merged and constitute a new cell. This may be caused by: (1) the high pulse density in the ESM environment, which results in the time overlapping of received pulses, (2) the inaccuracy in measuring the monopulse parameters of each intercepted pulse, which leads to the insertion of a pulse into an incorrect cell. In [7], we exploit the difference in the histogram bin distribution between the ideal and the real deinterleaving to calculate a confidence level for each cell.

The separation in the AOA between radars in the instantaneous view of the ESM should be wide enough to allow the adaptive deinterleaving algorithm to be applied properly. In modern warfare, some radars may have AOA close to each other. Therefore, the pulses emitted from these radars will be merged and constitute a single cell. Consequently, the confidence level representing the quality of this cell will be very low and the identification of the radars in this cell will not be feasible. To overcome this problem, we propose a new joint deinterleaving/identification approach. This approach will be applied to cells with a low 
confidence level and will allow the ESM system to identify the radars with very close AOAs and their pulses merged in a single cell.

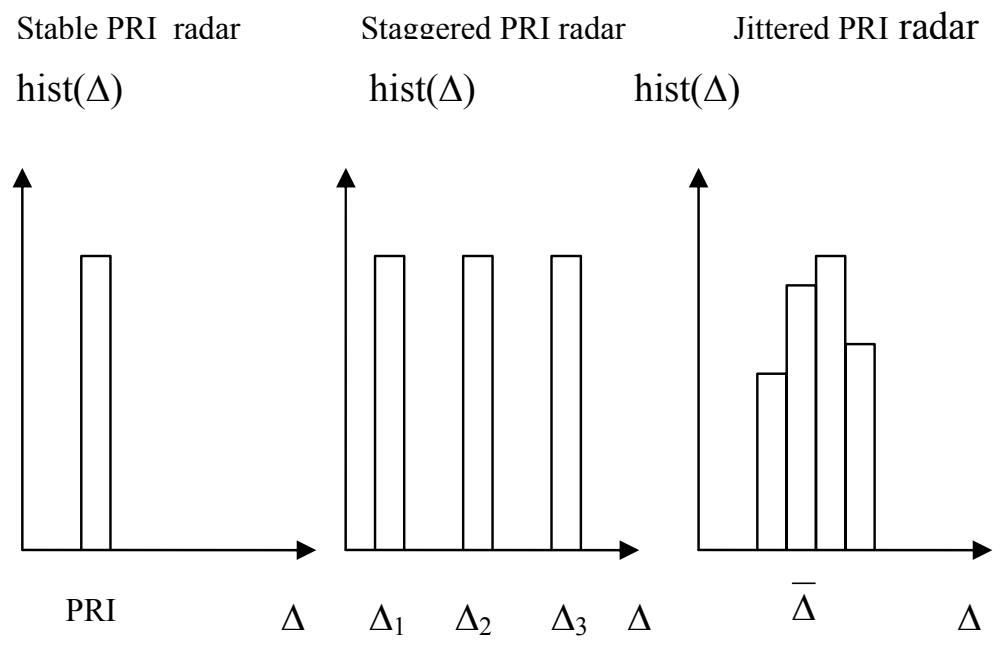

Fig. 1. $\Delta$-Histogram of different PRI radars

\section{JOINT DEINTERLEAVING IDENTIFICATION USING MATRIX DIFFERENCES}

This Section proposes a technique that uses the concept of joint deinterleaving/identification to deinterleave the intercepted pulses and identify their corresponding radars. The proposed method can detect the presence of the test radar and extract its corresponding pulses. The final objective of deinterleaving is emitter identification. The proposed approach will perform deinterleaving through identification, thus accomplishing both tasks in a single step. The input data of the approach proposed in this paper is the TOAs of the pulses belonging to a single radar cell that contains pulses from more than one radar having the same $A O A$.

Deinterleaving of pulses inside the received stream using the conventional method in [8] requires calculating the TOA differences between each pulse and all successive pulses in the received stream. Calculating these differences requires large number of computations, which is directly proportional to the square of the number of pulses in the received stream $\left(N^{2} / 2\right)$. Although, the generated differences include the PRIs of all radars present in the received stream, it is difficult to distinguish between the TOA differences corresponding to correct $P R I$ values and the other differences corresponding to multiple sums and differences of $P R I$ values. The detection of staggered PRI radars or pseudo random PRI radars is also not feasible using the conventional methods [8].

Thus, we propose a new technique like the conventional method [8] estimates the PRIs of the radars presented in the environment, but overcomes the main two drawbacks of the conventional method [8]. Firstly it generates the differences between each pulse and all successive pulses in the received stream without requiring a large number of computations. Secondly, it avoids the generation of false radars by applying the concept of joint deinterleaving/identification.

The proposed technique saves the indices of the pulse pair that produces each TOA difference. Then, by linking the indices of each pulse pair that produces the specific PRI values, we can 
identify the pulses that belong to the test radar. In the case of the pseudo random PRI radar, we link the pulse indices of all pulse pairs whose TOA differences produce one of the PRI values that the pseudo random PRI radar uses.

To make the computations efficient, the proposed method uses matrix operations to generate the TOA differences between each pulse and all successive pulses in the received stream and at the same time save the indices of each pulse pair that produces the TOA differences. It should be noted that a matrix operation does not actually reduce the hardware complexity. However, in software, a matrix operation is much more efficient because "FOR" loops are not required. Hence, reducing the overhead associated with these loops. Using MATLAB version 12, the simulation times for generating the differences between each pulse and all successive pulses as well as saving the values of these differences by applying the matrix differences are reduced approximately by 264 times and by 225 times with MATLAB version 13.

The TOA difference matrix is generated as follows. Suppose that during the given observation time interval $\theta, N$ pulses are intercepted. Then, from the TOAs of these $N$ intercepted pulses, we generate two $N \times N$ matrices. The first matrix is denoted TOAR: the $i^{\text {th }}$ row of this matrix consists of $N$ elements, all are equal to the TOA of the $i^{\text {th }}$ pulse. The second matrix is denoted TOAC: the $j^{\text {th }}$ column of this matrix consists of $N$ elements, all are equal to the TOA of the $j^{\text {th }}$ pulse. Subtracting the TOAC matrix from the TOAR matrix generates the TOAD matrix. This matrix subtraction is done using MATLAB and generates the TOA differences between each pulse and all successive pulses in the received stream in one step. Fig. 2 presents the TOAR, $T O A C$, and TOAD matrices.

\begin{tabular}{|c|c|c|c|c|c|c|}
\hline$C_{1}$ & $C_{2}$ & $C_{3}$ & & & $C_{N-1}$ & $C_{N}$ \\
\hline $\mathrm{TOA}_{1}$ & $\mathrm{TOA}_{1}$ & $\mathrm{TOA}_{1}$ & $\mathrm{TOA}_{1}$ & $\mathrm{TOA}_{1}$ & $\mathrm{TOA}_{1}$ & $\mathrm{TOA}_{1}$ \\
\hline $\mathrm{TOA}_{2}$ & $\mathrm{TOA}_{2}$ & $\mathrm{TOA}_{2}$ & $\mathrm{TOA}_{2}$ & $\mathrm{TOA}_{2}$ & $\mathrm{TOA}_{2}$ & $\mathrm{TOA}_{2}$ \\
\hline $\mathrm{TOA}_{3}$ & $\mathrm{TOA}_{3}$ & $\mathrm{TOA}_{3}$ & $\mathrm{TOA}_{3}$ & $\mathrm{TOA}_{3}$ & $\mathrm{TOA}_{3}$ & $\mathrm{TOA}_{3}$ \\
\hline. &. &. &. & . &. &. \\
\hline . & . & . & . & . & . & . \\
\hline . & . & . & . & . & . & . \\
\hline $\mathrm{TOA}_{N-2}$ & $T O A_{N-2}$ & $\mathrm{TOA}_{N-2}$ & $\mathrm{TOA}_{N-2}$ & $T O A_{N-2}$ & $T^{2} A_{N-2}$ & $T O A_{N-2}$ \\
\hline $\mathrm{TOA}_{N-1}$ & $T_{O A} A_{N-1}$ & $\mathrm{TOA}_{N-1}$ & $T O A_{N-1}$ & $T A_{N-1}$ & $T A_{N-1}$ & $T O A_{N-1}$ \\
\hline$T O A_{N}$ & $T O A_{N}$ & $\mathrm{TOA}_{N}$ & $T O A_{N}$ & $T O A_{N}$ & $T O A_{N}$ & $T O A_{N}$ \\
\hline
\end{tabular}

The TOAR matrix

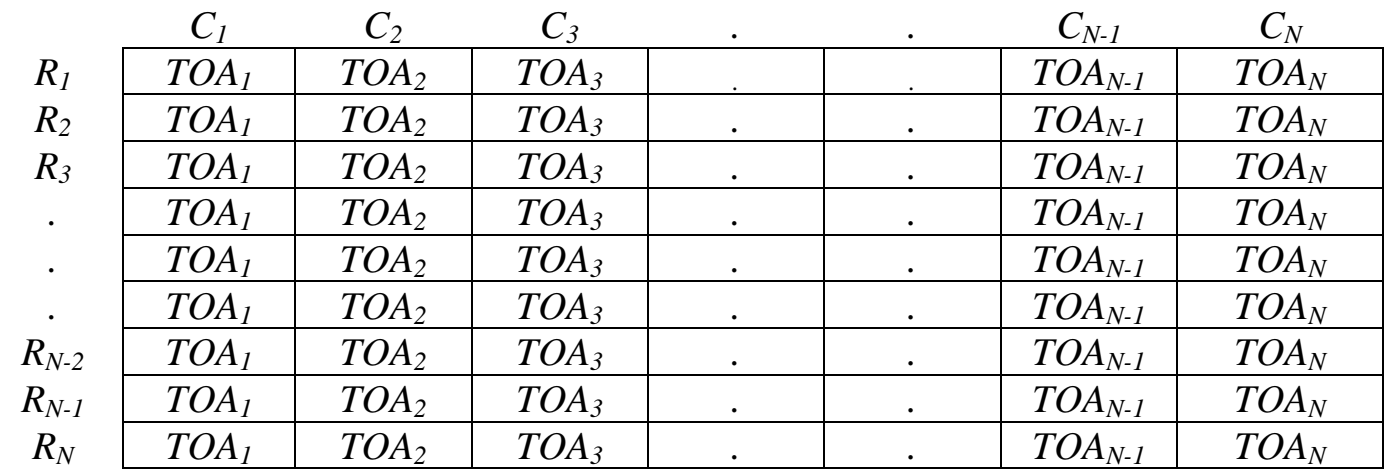

The TOAC matrix 


\begin{tabular}{|c|c|c|c|c|c|c|c|c|}
\hline & $C_{1}$ & $C_{2}$ & $C_{3}$ & & & $C_{N-2}$ & $C_{N-1}$ & $C_{N}$ \\
\hline$R_{1}$ & $T O A D_{1,1}$ & $T O A D_{1,2}$ & $T O A D_{1,3}$ & & & $T O A D_{1, N-2}$ & $T O A D_{1, N-1}$ & $T O A D_{1, N}$ \\
\hline$R_{2}$ & $T O A D_{2,1}$ & $T O A D_{2,2}$ & $T O A D_{2,3}$ & . & . & $T O A D_{2, N-2}$ & $T O A D_{2, N-1}$ & $T O A D_{2, N}$ \\
\hline$R_{3}$ & $\mathrm{TOAD}_{3,1}$ & $T O A D_{3,2}$ & $T O A D_{3,3}$ & - & & $\mathrm{TOAD}_{3, N-2}$ & $\mathrm{TOAD}_{3, N-1}$ & TOAD $_{3, N}$ \\
\hline . & . & 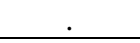 & & . & & . & . & \\
\hline . & . & . & . & . & & . & . & $\dot{-}$ \\
\hline & TOAD & & TOA & & & & & TOA \\
\hline $\begin{array}{l}K_{N-2} \\
R_{N-1}\end{array}$ & $T O A D_{N-1,1}$ & $T O A D_{N-2,2}$ & $T O A D_{N-1,3}$ & · & · & $T O A D_{N-1, N-2}$ & $T O A D_{N-2, N-1}$ & $T O A D_{N-2, N}$ \\
\hline$R_{N}$ & $T O A D_{N, 1}$ & $T O A D_{N, 2}$ & $T O A D_{N, 3}$ & • & & $T O A D_{N, N-2}$ & $T O A D_{N, N-1}$ & $T O A D_{N, N}$ \\
\hline
\end{tabular}

The TOAD matrix; TOAD $D_{i, j}=$ TOA $_{i}-T_{O A A_{j}}$

Fig. 2. The TOAR, TOAC, and the TOAD matrices

It is clear that the upper right triangle of the TOAD difference matrix is the negative of the lower left triangle. The main diagonal $\left(T O A D_{i},_{i}=T O A_{i}-T O A_{i} ; 1 \leq i \leq N\right)$ is the TOA difference between every pulse and itself. Hence, all its elements are zeros. The diagonal $\left(T O A D_{i, i-1}=T O A_{i}-T O A_{i-1} ; 2 \leq i \leq N\right)$ consists of the $T O A$ difference between every pulse and its next pulse, and the diagonal $\left(T O A D_{i, i-2}=T O A_{i}-T O A_{i-2} ; 3 \leq i \leq N\right)$ consists of the TOA difference between every pulse and the second next pulse, and so forth. We process only positive elements of the TOAD matrix, i.e., only the elements in the lower left triangle.

The coordinates of any element in the TOAD matrix give the pulse indices of the pulse pair, which produces the TOA difference stored in this element. For example the element of the $T O A D$ matrix at the eighth row and the fourth column is the TOA difference between the $8^{\text {th }}$ pulse and the $4^{\text {th }}$ pulse. The column index of any TOAD element gives the index of the start pulse in the pulse pair that generates this TOAD element and the row index gives the index of the end pulse in the pulse pair. By linking the pulse indices corresponding to the pulse pairs that match the PRIs of the test radar, we can identify the indices of all pulses belonging to the test radar.

The next section shows how we link the pulse indices to identify the pulses belonging to the test radar.

\section{EXTRACTION OF PULSES BELONG TO THE IDENTIFIED RADARS}

For clarity, we summarize the pertinent operations. Let an element of the TOAD be $\mathbf{T O A D _ { i , j }}$ which is the result of the TOA difference between pulse $i$ and pulse $j ; i$ refers to the index of the end pulse in the pair $(i, j)$ and $j$ refers to the index of the start pulse in the pair $(i, j)$,

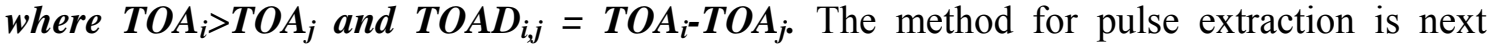
described.

After generating the TOAD matrix, we generate two vectors. The first vector is a $1 \times N$ row vector aligned parallel to the first row of the TOAD matrix. The elements of this vector are all zeros and the elements corresponding to the indices of the start pulse in each pulse pair whose TOA difference matches one of the PRI of the test radar will be changed to one. The second vector is an $N \times 1$ column vector aligned parallel to the first column of the TOAD matrix. The elements of this vector are all zeros and the elements corresponding to the indices of the end pulse in each pulse pair whose TOA difference matches one of the PRI of the test radar will be 
changed to one. Hence, the elements of the TOAD matrix that match one of the PRIs of the test radar will lead to an insertion of 1's in the corresponding places of the row and column vectors. The identification of the pulses belonging to the test radar is presented in Fig. 3.

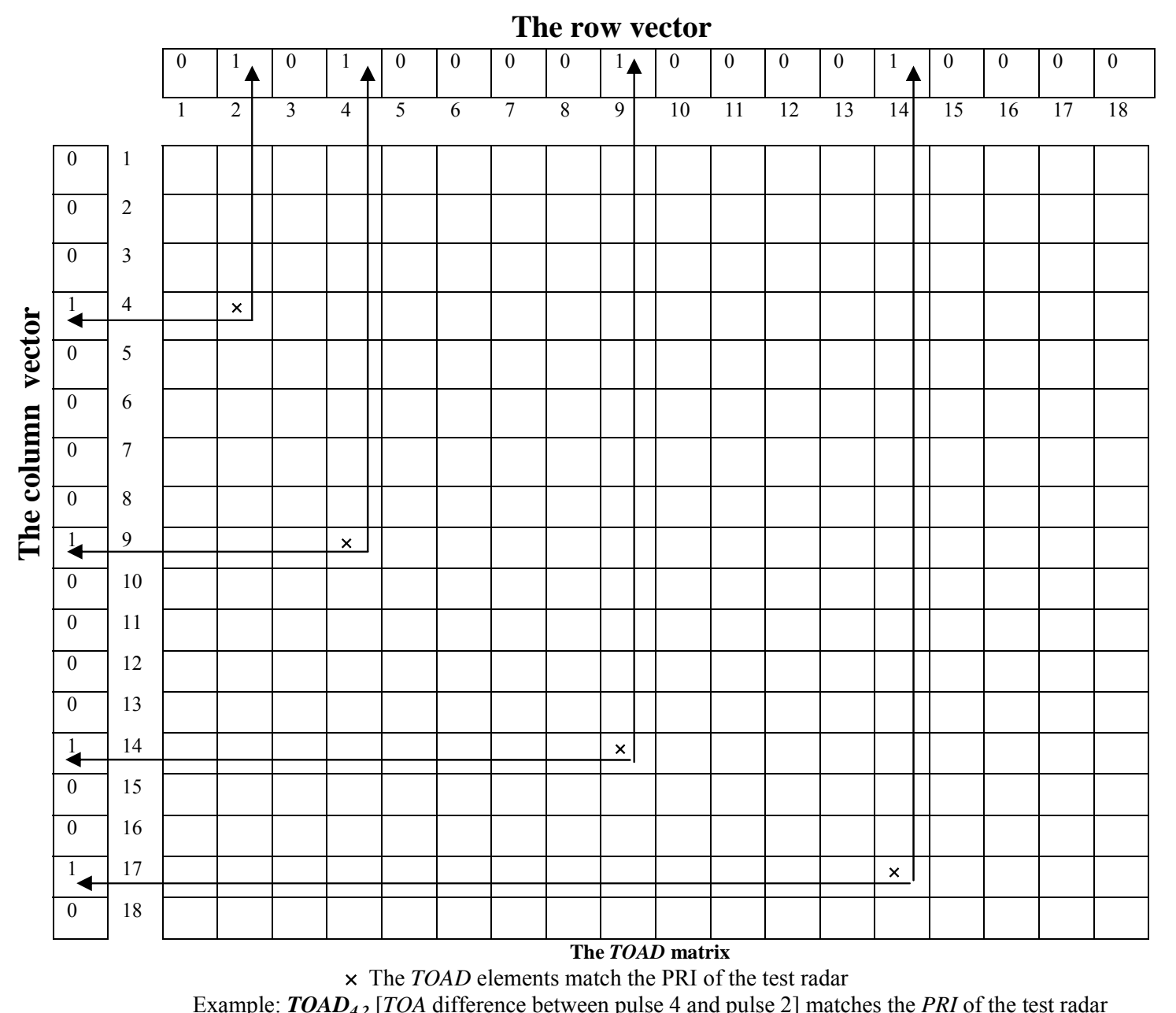

Fig. 3. The TOAD matrix and the corresponding row and column vectors

As seen in Fig. 3, the row and column vectors have 1's in the places corresponding to the $T O A D$ elements that match the PRI of the test radar. Only pairs with an index of the start pulse equal to or larger than the index of the end pulse of the previous matching pair are checked. As a consequence, for any two successive matching pairs, the index of the start pulse in the second pair must be larger than or equal to the index of the end pulse in the first pair. The index of the start pulse in the second pair is equal to the index of the end pulse in the first pair if there is no missing and no extraneous pulse and the index of the start pulse in the second pair is larger than the index of the end pulse in the first pair otherwise.

As an example the two successive pairs $(4,2)$ and $(9,4)$ in Fig. 3 , satisfy the above condition. Because the index of the start pulse in the second pair is 4 , which equal to the index of the end pulse in the first pair. This means that pulses 2, 4 and 9 belong to the test radar and no pulse of that radar is missing between pulses 2 and 9. Fig. 3 presents the row and the column vectors that are obtained when applying the proposed method to the TOAD matrix in Fig. 3. 
(a) The row vector

\begin{tabular}{|l|l|l|l|l|l|l|l|l|l|l|l|l|l|l|l|l|l|}
\hline 0 & 1 & 0 & 1 & 0 & 0 & 0 & 0 & 1 & 0 & 0 & 0 & 0 & 1 & 0 & 0 & 0 & 0 \\
\hline
\end{tabular}

(b) The column vector

\begin{tabular}{|l|l|l|l|l|l|l|l|l|l|l|l|l|l|l|l|l|l|}
\hline 0 & 0 & 0 & 1 & 0 & 0 & 0 & 0 & 1 & 0 & 0 & 0 & 0 & 1 & 0 & 0 & 1 & 0 \\
\hline
\end{tabular}

(c) column OR row

\begin{tabular}{|l|l|l|l|l|l|l|l|l|l|l|l|l|l|l|l|l|l|}
\hline 0 & 1 & 0 & 1 & 0 & 0 & 0 & 0 & 1 & 0 & 0 & 0 & 0 & 1 & 0 & 0 & 1 & 0 \\
\hline
\end{tabular}

Fig. 4. OR'ing of the row and column vectors

OR'ing the row and the column vectors yields a vector shown in Fig. 4(c) whose elements are equal to 1 in the places corresponding to the indices of the pulses belonging to the test radar. Thus, the number of ' 1 's in this vector represents the actual number of pulses, $N_{P}$, belonging to the test radar, in the received stream.

For every test radar, the number of pulses belonging to that radar, is found using the above method and this number is compared with the number of pulses assumed to be emitted from that radar under the ideal situation, $N_{\text {Exact }}$, i.e., when there are no missing pulses. The ratio between the two values is the Fig. of merit of the test radar, F. If the Fig. of merit is larger than or equal to the accepted value of the factor of successful processing of the ESM system $F_{s}$, the test radar is said to be an identified radar and its pulses will be extracted from the received stream. On the other hand, if the Fig. of merit is less than the accepted value of $F_{s}$ then this indicates that the test radar is not present in the received stream.

It is worth noting that an acceptable value of $F_{S}$ (the factor of successful processing of the ESM system) depends on of the PRI of the test radar. The factor of successful processing is the ratio of the received pulses that are successfully deinterleaved and assigned to their corresponding radar cells. The theoretical derivation of $F_{s}$ is given in [9]. The number of pulses $N_{\text {Exact }}$ of the test radar in the ideal case is given by

$$
N_{\text {Exact }} \approx L \times\left[\frac{T_{\text {Obs }}}{P R I_{\text {Frame }}}\right]
$$

where $L$ is the number of $P R I$ values the test radar uses, $P R I_{\text {Frame }}$ is the sum of the $P R I$ values of the test radar and $T_{O b s}$ is the given observation time. The Fig. of merit $F$ of the test radar is defined as

$$
F=\frac{N_{P}}{N_{\text {Exact }}}
$$

$F$ is then compared with the threshold $T$ that is given by 


$$
T \cong F_{s}
$$

where $F_{s}$ is the factor of successful processing given in [9].

If $F$ is larger than or equal to the threshold, we decide that the test radar is present. Consequently, the pulses of the test radar will be extracted from the received pulse stream. The number of missing pulses from the test radar is the difference between the $N_{P}$ and $N_{\text {Exact }}$.

After each successful identification, some elements of the TOAD matrix will not be checked in the next identification. Hence, the required number of computations will be reduced. The elements of the TOAD matrix that will not be checked are those elements whose row or column index equals one of the pulse indices of an identified radar. For example in Fig. 3, pulses 2, 4, 9, 14 and 17 belong to an identified radar. Then, all the elements of the rows and the columns number 2,4,9,14 and 17 of the TOAD matrix will not be checked again in future identifications as shown in Fig. 5.

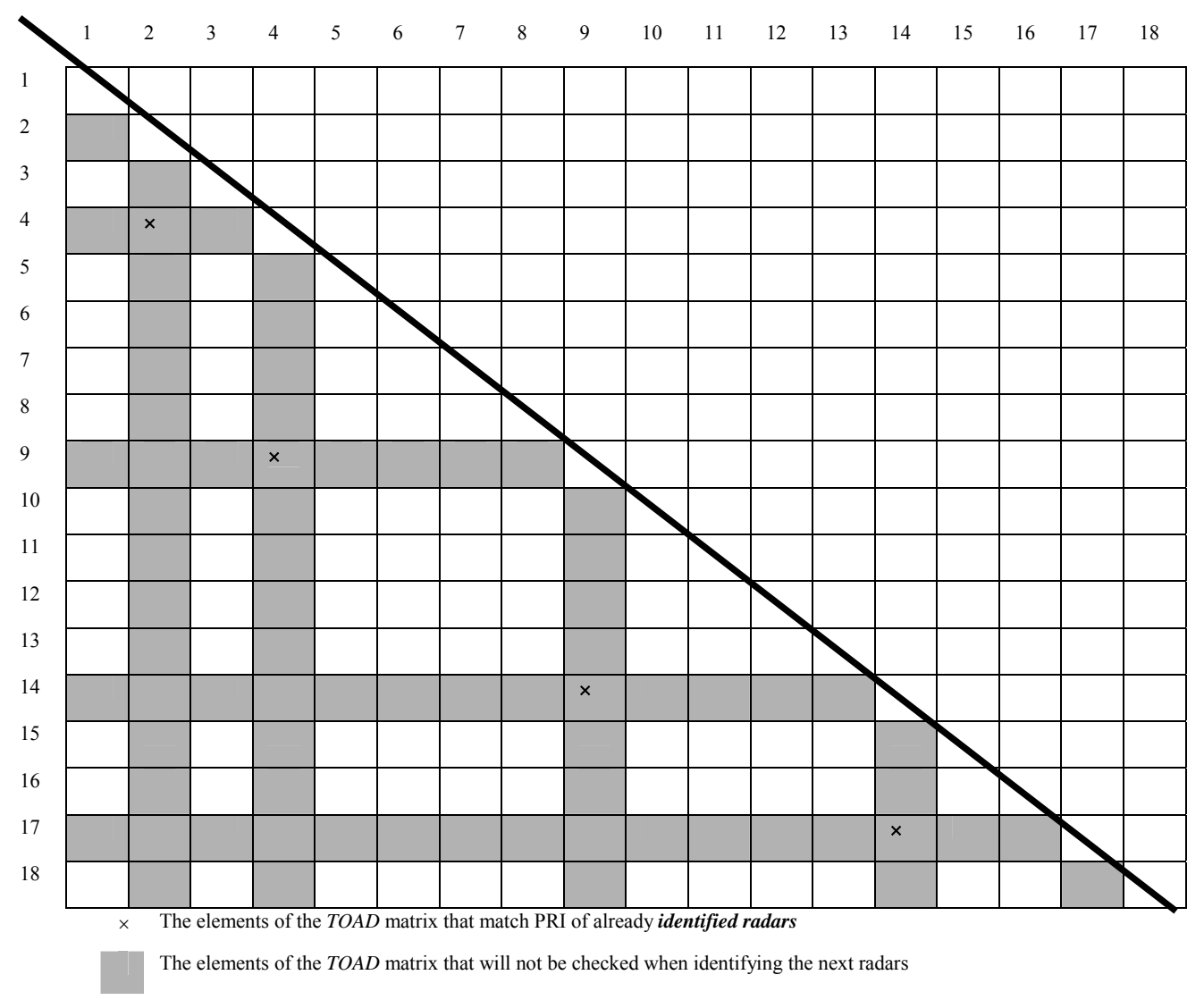

Fig. 5. The reduction of the TOAD elements needed for identification of next radars

As seen in Fig. ${ }^{\circ}$, many elements of the TOAD matrix will not be checked when identifying the next radars. Hence, there is saving in computations after each identification. The amount of savings depends on the number of pulses in the identified radar. As discussed in Section $r$, we are only processing the lower left triangle of the TOAD matrix. In the following section, we present the simulations results of the proposed method. 


\section{COMPUTER SIMULATION}

In this section, the proposed technique is used to deinterleave and identify three radars with the same $A O A$.

Table 1. Application of the joint deinterleaving / identification using matrix difference to identify intercepted radars. $T_{\text {Obs }}=0.165 \mathrm{sec}$., $T_{s}=10 \mu \mathrm{sec}, T_{a}=50 \mu \mathrm{sec}, F_{\mathrm{s}}=$ 0.88 .

\begin{tabular}{|c|c|c|c|}
\hline & PRI Mode & $\begin{array}{c}\text { Fig. of } \\
\text { Merit }\end{array}$ & $\begin{array}{c}\text { Missing } \\
\text { Pulses }\end{array}$ \\
\hline Radar 1 & $\begin{array}{c}\text { Stable } P R I \\
P R I=952 \mu \mathrm{sec}\end{array}$ & 0.915 & 14 \\
\hline Radar 2 & $\begin{array}{c}\text { Pseudo Random } P R I \\
\mathrm{P}(P R I=862 \mu \mathrm{sec})=0.332 \\
\end{array}$ & 0.901 & 37 \\
& $\mathrm{P}(P R I=287 \mu \mathrm{sec})=0.346$ \\
& $\mathrm{P}(P R I=172 \mu \mathrm{sec})=0.320$ & & \\
\hline Radar 3 & $\begin{array}{c}\text { Jittered } P R I \\
P R I=909 \mu \mathrm{sec} \\
\mathrm{JW}=30 \mu \mathrm{sec}\end{array}$ & 0.885 & \\
\hline $\begin{array}{c}\text { Radar 4 } \\
\text { [Not present in the } \\
\text { received stream }]\end{array}$ & $\begin{array}{c}\text { Stable } P R I \\
\text { PRI }=1024 \mu \mathrm{sec}\end{array}$ & 0.071 & 150 \\
\hline
\end{tabular}

It is clear from Table ', that the proposed technique can successfully deinterleave the intercepted pulses and identify their corresponding radars. It is also clear that the proposed method can successfully identify stable, jittered and staggered (pseudo random) $P R I$ radars with the same AOA. The proposed method calculates the Fig. of merit and the number of missing pulses for each test radar. Hence, it can successfully detect that the test radar is not present in the received stream as seen in the case of the radar 4, which has a very low Fig. of merit.

In summary. By applying this concept the proposed method can identify stable, jittered and staggered (pseudo-random) PRI radars and at the same time determine that some of the test radars are not present in the received stream.

\section{CONCLUSIONS}

A joint deinterleaving/identification approach using matrix differences has been described and analyzed in this paper. The main advantages of the proposed method in this paper are as follows. Firstly, it can generate the TOA differences between every pulse and all successive pulses in the received stream using one matrix operation. Hence, instead of requiring intensive looping to subtract the TOA of successive pulses element by element it uses instead a matrix operation. This makes the computations more efficient. The second advantage of the proposed method is the application of the concept of joint deinterleaving/identification The proposed approach is applied to identify radars having very close AOA and their pulses are merged into a single cell. The simulation results show that the proposed approach can be successfully applied to identify stable, jittered, and staggered PRI radars. Moreover, the proposed approach 
could be applied as an integral part of the adaptive deinterleaving algorithm. Thus, the ESM system can take appropriate actions against the identified radars and avoid wasting available resources against false radars.

\section{REFRENCES}

[1] SCHLEHER, D., Introduction to Electronic Warfare, Artech House, Inc., 1982.

[2] MARDIA, H. K., "New Techniques for Deinterleaving Repetitive Sequences," IEE Proc. F, Comm., Radar \& Signal Processing, 136, (4), pp. 149-154, 1989.

[3] MILOJEVIC, D. J., and POPOVIC, B. M., "Improved Algorithm for Deinterleaving of Radar Pulses," IEE Proc. F, Comm., Radar \& Signal Processing, 139, (1), pp. 98-104, 1992.

[4] MOORE, J. B. and KRISHNAMURTHY, V., “ Deinterleaving of Pulse Trains Using Discrete-Time Stochastic Dynamic Linear Models," IEEE Transactions on Signal Processing, Vol. 42, No. 11, Nov. 1994.

[5] WILKINSON, D. R., and WATSON, A. W., " Use of Metric Techniques in ESM Data Processing," IEE Proc. F, Comm., Radar \& Signal Processing, 132, (7), pp. 621-625, 1985.

[6] CHAN, Y. T., CHAN, F., and HASSAN, H. E. ABOU-BAKR, "Performance Evaluation of ESM Deinterleaving Using TOA Analysis," The $14^{\text {th }}$ International Conference on Microwave, Radar and Wireless Communications, Vol. 2, pp. 341-350, Poland, May 2002.

[7] HASSAN, H. E. ABOU-BAKR, CHAN, F., and CHAN, Y. T., "Adaptive Deinterleaving Algorithm in Dense ESM System Environments," The Third International Conference on Electrical Engineering, Egypt, May 2002.

[8] WILEY, R. G., The Analysis of Radar Signals, Artech House, 1993.

[9] HASSAN, H. E. ABOU-BAKR, CHAN, F., and CHAN, Y. T, "Queueing Analysis of the Deinterleaving of Radar Pulses in a Dense Emitter Environment," the IEEE Canadian Conference on Electrical and Computer Engineering, CCECE 2003, Montréal, May 2003. 